\title{
Efeito de intervenções aquáticas sobre os sintomas da fibromialgia: uma revisão
}

\author{
Effect of aquatic interventions on the symptoms of fibromyalgia: a review \\ Efecto de las intervenciones acuáticas sobre los síntomas de fibromialgia: una revisión
}

Recebido: 07/11/2021 | Revisado: 16/11/2021 | Aceito: 17/11/2021 | Publicado: 28/11/2021

\author{
Leonardo De Los Santos Bloedow \\ ORCID: https://orcid.org/0000-0003-0481-5808 \\ Universidade Federal do Rio Grande do Sul, Brasil \\ E-mail: leobloedow@gmail.com \\ Priscilla Cardoso da Silva \\ ORCID: https://orcid.org/0000-0002-5033-6878 \\ Universidade Federal do Rio Grande do Sul, Brasil \\ E-mail: priscilla.cardoso@ufrgs.br \\ Patrick dos Santos Guimarães \\ ORCID: https://orcid.org/0000-0003-0562-4165 \\ Universidade Federal do Rio Grande do Sul, Brasil \\ E-mail: ps.guimaraes89@gmail.com
}

\begin{abstract}
Resumo
Introdução: Síndrome da fibromialgia é uma condição idiopática crônica de dor musculoesquelética generalizada que afeta principalmente as mulheres. Objetivo deste estudo é investigar na literatura os efeitos de intervenções aquáticas na fibromialgia em relação a parâmetros físicos e psicológicos, e os principais métodos de avaliação. Métodos: Foi realizada uma pesquisa bibliográfica nos bancos de dados Embase, PubMed e Scielo com os descritores: Fibromyalgia, Fibromyalgia Syndrome, Aquatic exercise e Water exercise, totalizando 23 artigos. Resultados: Terapias aquáticas são recomendadas como tratamento pelos seus benefícios. A imersão na água permite a reprodução de movimentos de maneira segura e variada minimizando os impactos quando comparados aos exercícios no solo. Pacientes com fibromialgia relatam a sensação de relaxamento e alívio dos sintomas, após a prática de atividade em meio aquático. Conclusão: Intervenções aquáticas apresentam-se como recursos terapêuticos eficientes para o tratamento da dor e melhora da qualidade de vida de pessoas com fibromialgia.
\end{abstract}

Palavras-chave: Fibromialgia; Exercício físico; Terapia por exercício; Reabilitação.

\begin{abstract}
Introduction: Fibromyalgia syndrome is a chronic idiopathic condition of generalized musculoskeletal pain that mainly affects women. The aim of this study is to investigate in the literature the effects of aquatic interventions in fibromyalgia in relation to physical and psychological parameters, and the main assessment methods. Methods: A literature search was carried out in Embase, PubMed and Scielo databases with the descriptors: Fibromyalgia, Fibromyalgia Syndrome, Aquatic exercise and Water exercise, total of 23 articles. Results: Aquatic therapies are recommended as a treatment for their benefits. Immersion in water allows the reproduction of movements in a safe and varied way, minimizing impacts when compared to exercises on the ground. Fibromyalgia patients report the sensation of relaxation and relief of symptoms, after the practice of activity in an aquatic environment. Conclusion: Aquatic interventions are presented as efficient therapeutic resources to treat pain and improve the quality of life of people with fibromyalgia.
\end{abstract}

Keywords: Fibromyalgia; Exercise; Exercise therapy; Rehabilitation.

\section{Resumen}

Introducción: El síndrome de fibromialgia es una condición idiopática crónica de dolor musculoesquelético generalizado que afecta principalmente a mujeres. El objetivo de este estudio es investigar en la literatura los efectos de las intervenciones acuáticas en la fibromialgia en relación con los parámetros físicos y psicológicos, y los principales métodos de evaluación. Métodos: Se realizó una búsqueda bibliográfica en las bases de datos Embase, PubMed y Scielo con los descriptores: Fibromialgia, Síndrome de Fibromialgia, Ejercicio acuático y Ejercicio acuático, totalizando 23 artículos. Resultados: Se recomiendan las terapias acuáticas como tratamiento por sus beneficios. La inmersión en agua permite la reproducción de movimientos de forma segura y variada, minimizando los impactos respecto a los ejercicios en el suelo. Los pacientes con fibromialgia refieren la sensación de relajación y alivio de los síntomas, luego de la práctica de actividad en un ambiente acuático. Conclusión: Las intervenciones acuáticas se presentan como recursos terapéuticos eficientes para tratar el dolor y mejorar la calidad de vida de las personas con fibromialgia.

Palabras clave: Fibromialgia; Ejercicio Físico; Terapia por ejercicio; Rehabilitación. 


\section{Introdução}

A fibromialgia é uma síndrome caracterizada pela dor crônica difusa no corpo e a presença de pontos de dor (denominados, tender points) em regiões específicas do corpo, conforme critérios definidos pelo Colégio Norte-Americano de Reumatologia (Wolfe et al., 2010). Além das queixas dolorosas, vários outros sintomas estão presentes, envolvendo estruturas não pertencentes ao aparelho locomotor, entre elas estão as alterações do sono, cefaleia, parestesia em membros superiores e inferiores, fadiga, queixas gastrintestinais e geniturinárias. Outros sintomas descritos são as alterações psicológicas, como a depressão, níveis elevados de somatização e ansiedade, insatisfação e modificação da percepção do estado de saúde (Martinez et al., 2017; Theoharides et al., 2015).

Esta síndrome acomete pessoas de ambos os sexos, porém há uma clara associação com o sexo feminino, correspondendo a $90 \%$ dos casos. A média de idade dos pacientes está ao redor da quinta década de vida (Souza \& Perissinotti 2018).

A fisiopatologia da fibromialgia parece ser tão incerta e multicausal quanto a sua etiologia. Todavia, a importância de fatores sociais, emocionais, familiares, aliados a uma característica de maior resposta aos estímulos dolorosos, o baixo nível de condicionamento físico são as hipóteses mais plausíveis. Explicações com modelos multifatoriais permitem o entendimento dessa síndrome como a soma de desordens que acabam se manifestando pela associação de variados sintomas (Talotta et al., 2017; Theoharides et al., 2015).

O tratamento tradicional da fibromialgia é através do uso de fármacos como analgésicos e antidepressivos, aliados à educação do paciente com intuito de fornecer o melhor esclarecimento sobre a doença sintomas (Vilaça et al., 2020; Talotta et al., 2017).

Tendo em vista que nem todos pacientes respondem favoravelmente unicamente aos tratamentos farmacológicos, é importante considerar os efeitos de métodos terapêuticos multimodais sobre os sintomas da fibromialgia. Exercícios físicos realizados em meio aquático mostram-se na literatura como uma opção de sucesso, obtendo resultados favoráveis para a melhora dos sintomas da fibromialgia (Kümpel et al, 2020; Letieri et al., 2013).

Assim o presente estudo tem como objetivo abordar os principais efeitos associados a intervenções com exercícios aquáticos sobre os sintomas da fibromialgia bem como referir brevemente o modelo fisiopatológico e os principais instrumentos de avaliação dos sintomas desta síndrome.

\section{Metodologia}

O presente estudo é caracterizado como uma revisão de literatura, do tipo narrativa e discursiva, que abordará o tema: Os principais efeitos da prática de exercícios físicos realizados na água sobre os sintomas da fibromialgia e apresentar os principais instrumentos de avaliação do impacto na saúde e bem estar dos pacientes com fibromialgia.

Foi realizada uma pesquisa bibliográfica nos bancos de dados dos sistemas Embase, National Library of Medicine (PUBMED) e a Scientific Electronic Library Online (Scielo) com os seguintes descritores em língua inglês: “Fibromyalgia, Fibromyalgia Syndrome, Aquatic exercise and Water exercise”, adendo os booleanos 'OR" e 'AND'. A pesquisa online dos artigos limitou-se aos idiomas inglês, português e espanhol publicados no período de 2001 a 2018 referentes ao tema.

Primeiramente foram encontrados 309 artigos nos bancos de dados, após exclusão das duplicatas e leitura dos títulos e resumos, foram selecionados 47 artigos para leitura completa. Sendo selecionados para este estudo 22 artigos diretamente sobre fibromialgia e 1 estudo de validação. Por se tratar deste modelo de revisão, não houve a avaliação da qualidade dos estudos, segundo Quadro 1 (abaixo) demonstra-se a síntese das referências utilizadas neste estudo. 
Quadro 1: Síntese dos artigos sobre fibromialgia incluídos na presente revisão.

\begin{tabular}{|c|c|c|c|c|}
\hline Autores & População & Estudo/Instrumentos & Intervenção & Desfecho \\
\hline $\begin{array}{l}\text { Wolfe et al., } \\
2010\end{array}$ & $\mathrm{~N}=829$ & $\begin{array}{l}\text { Estudo Multicêntrico. } \\
\text { Instrumentos: No pontos gatilhos } \\
\text { (Tender points); Índice de dor } \\
\text { generalizado (WPI) e Escala de } \\
\text { Gravidade dos Sintomas (SS). }\end{array}$ & $\begin{array}{l}\text { Medidas de avaliação; } \\
\text { 4meses }\end{array}$ & $\begin{array}{c}\text { Das avaliações } 88,1 \% \text { pacientes } \\
\text { satisfizeram os critérios de } \\
\text { classificação do American } \\
\text { College of Rheumatology (ACR). } \\
\text { A SS permite a avaliação da } \\
\text { gravidade dos sintomas de } \\
\text { fibromialgia. }\end{array}$ \\
\hline $\begin{array}{l}\text { Martinez JE } \\
\text { et al., } 2017\end{array}$ & $\mathrm{~N}=810$ & $\begin{array}{c}\text { Estudo transversal coorte } \\
\text { multicêntrico. Instrumentos: } \\
\text { Fibromyalgia Impact } \\
\text { Questionnaire (FIQ), } \\
\text { Polysymptomatic Distress Scale } \\
\text { (PDS) }\end{array}$ & & $\begin{array}{c}\text { Houve correlação moderada entre } \\
\text { o monitoramento pelo FIQ e PDS } \\
\text { e a maior parte dos pacientes } \\
\text { manteve-se estável. }\end{array}$ \\
\hline $\begin{array}{l}\text { Talotta } R \text { et } \\
\text { al., } 2015\end{array}$ & & Revisão de literatura & $\begin{array}{l}\text { Artigos publicados de } \\
\text { jan/2016 a jan/2017 } \\
\text { relacionados à } \\
\text { etiopatogênese, avaliação } \\
\text { e tratamento da } \\
\text { fibromialgia }\end{array}$ & $\begin{array}{c}\text { A avaliação deve ser global com o } \\
\text { intuito de detectar a prevalência } \\
\text { do fenótipo e intervenção mais } \\
\text { apropriada. }\end{array}$ \\
\hline $\begin{array}{l}\text { Theoharis C } \\
\text { et al., } 2015\end{array}$ & & Revisão de literatura & $\begin{array}{l}\text { Artigos relacionados à } \\
\text { patogênese }\end{array}$ & $\begin{array}{l}\text { Tratamento deve ser com por uma } \\
\text { abordagem multidimensional } \\
\text { (educação ao paciente, terapia } \\
\text { comportamental, exercícios e } \\
\text { controle da dor por fármacos), } \\
\text { para minimizar os efeitos } \\
\text { colaterais. }\end{array}$ \\
\hline $\begin{array}{l}\text { Heymann et } \\
\text { al., } 2017\end{array}$ & & Revisão sistemática & $\begin{array}{l}53 \text { estudos relacionados } \\
\text { ao diagnóstico da } \\
\text { fibromialgia }\end{array}$ & $\begin{array}{l}\text { Apresentação de nove } \\
\text { recomendações embasadas no } \\
\text { número de regiões dolorosas, } \\
\text { fadiga, sono e cognição e } \\
\text { sintomas somáticos. }\end{array}$ \\
\hline $\begin{array}{l}\text { Bidonde et } \\
\text { al., } 2014\end{array}$ & $\begin{array}{l}\mathrm{N}=881(866 \\
\text { mulheres e } 15 \\
\text { homens) }\end{array}$ & $\begin{array}{l}\text { Revisão sistemática e meta- } \\
\text { análise }\end{array}$ & $\begin{array}{l}16 \text { estudos sobre os } \\
\text { efeitos de programas de } \\
\text { treinamento aquático }\end{array}$ & $\begin{array}{l}\text { Há benefícios nos exercícios } \\
\text { aquáticos e terrestres, exceto na } \\
\text { força muscular (evidências de } \\
\text { qualidade muito baixa } \\
\text { favorecendo a terra). Nenhum } \\
\text { efeito adverso foi relatado. }\end{array}$ \\
\hline $\begin{array}{l}\text { Camargo RS; } \\
\text { Moser } \\
\text { ADDL; } \\
\text { Bastos LC; } \\
2014\end{array}$ & & Revisão de literatura & $\begin{array}{l}26 \text { estudos sobre a } \\
\text { abordagem dos métodos } \\
\text { avaliativos em } \\
\text { fibromialgia e a dor } \\
\text { crônica }\end{array}$ & $\begin{array}{l}\text { FIQ foi o mais citado entre os } \\
\text { estudos. Crescimento de } \\
\text { pesquisas na área }\end{array}$ \\
\hline $\begin{array}{l}\text { Izquierdo D, } \\
\text { \& Arrese A.; } \\
\quad 2007\end{array}$ & $\begin{array}{l}\mathrm{N}=60 \text { mulheres; } \\
\text { grupo fibromialgia } \\
(\mathrm{N}=35) \text { e controle } \\
(\mathrm{N}=25)\end{array}$ & $\begin{array}{l}\text { Ensaio clínico randomizado. } \\
\text { Instrumentos: Questionário sobre } \\
\text { estado de saúde, FIQ, } \\
\text { Localização dos Tender point; } \\
\text { Pittsburgh Sleep Quality Index } \\
\text { (PSQI); testes físicos; escala } \\
\text { psicológica (State Anxiety } \\
\text { Inventory- SAI), função cognitiva } \\
\text { (Paced Auditory Serial Addition } \\
\text { Task); e adesão. }\end{array}$ & $\begin{array}{l}\text { Programa de treinamento } \\
\text { aquático de } 16 \text { semanas } \\
\text { (força, resistência } \\
\text { aeróbica, e relaxamento) } \\
\text { realizados } 3 \text { vezes por } \\
\text { semana }\end{array}$ & $\begin{array}{c}\text { O grupo de exercícios teve } \\
\text { melhorias estatisticamente } \\
\text { significativas na pontuação FIQ e } \\
\text { em todos os testes } \\
\text { neuropsicológicos. }\end{array}$ \\
\hline $\begin{array}{l}\text { Perraton L, } \\
\text { Machotka Z, } \\
\text { Kumar S; } \\
2009\end{array}$ & & Revisão Sistemática & $\begin{array}{l}11 \text { estudos sobre } \\
\text { Programas de } \\
\text { hidroginástica usando } \\
\text { ensaios clínicos } \\
\text { randomizados }\end{array}$ & $\begin{array}{c}\text { Hidroterapia com exercícios } \\
\text { aeróbicos, períodos de } \\
\text { aquecimento e relaxamento são } \\
\text { comuns nos programas, auxilia na } \\
\text { melhoria da saúde mental. }\end{array}$ \\
\hline $\begin{array}{l}\text { Letieri RV et } \\
\text { al.; } 2013\end{array}$ & $\begin{array}{l}\mathrm{N}=64 \text { (mulheres; } \\
\text { grupo } \\
\text { hidrocinesioterapia } \\
(\mathrm{n}=33) \text { e grupo }\end{array}$ & $\begin{array}{l}\text { Ensaio Clínico Randomizado. } \\
\text { Instrumentos: Escala } \\
\text { Analógica Visual de Dor (EVA), }\end{array}$ & $\begin{array}{l}\text { Hidrocinesioterapia } 2 \\
\text { sessões de } 45 \text { minutos por } \\
\text { semana, total de } 30\end{array}$ & $\begin{array}{l}\text { Hidrocinesioterapia mostrou-se } \\
\text { eficaz como terapia para } \\
\text { fibromialgia. }\end{array}$ \\
\hline
\end{tabular}




\begin{tabular}{|c|c|c|c|c|}
\hline & controle $(n=31))$ & $\begin{array}{l}\text { o FIQ, e o Beck's Depression } \\
\text { Inventory (BDI) }\end{array}$ & sessões. & \\
\hline $\begin{array}{l}\text { Assis MR et } \\
\text { al; } 2006\end{array}$ & $\begin{array}{c}\mathrm{N}=60 \text { (mulheres } \\
\text { sedentárias; Grupo } \\
\text { Corrida água funda } \\
\text { (DWR) e } \\
\text { Exercícios } \\
\text { terrestres (LBE)). }\end{array}$ & $\begin{array}{c}\text { Ensaio Clínico Randomizado. } \\
\text { Instrumentos: Escala visual } \\
\text { analógica de dor, FIQ; BDI; } \\
\text { Short Form } 36 \text { Health Survey } \\
\text { (SF-36), e a avaliação global de } \\
\text { resposta do paciente a terapia } \\
\text { (PGART). }\end{array}$ & $\begin{array}{l}\text { Corrida água funda } \\
\text { (DWR) e Exercícios } \\
\text { terrestres (LBE) } 15 \\
\text { semanas de treinamento } \\
\text { no limiar anaeróbico. }\end{array}$ & $\begin{array}{l}\text { Ambos os grupos tiveram redução } \\
\text { na intensidade da dor. O DWR } \\
\text { mostrou vantagens na qualidade } \\
\text { de vida no âmbito emocional, no } \\
\text { FIQ e depressão. O ganho } \\
\text { aeróbico e a melhora dos } \\
\text { sintomas foram similares entre os } \\
\text { grupos. }\end{array}$ \\
\hline $\begin{array}{l}\text { Andrade SC } \\
\text { et al; } 2008\end{array}$ & & Revisão & $\begin{array}{c}8 \text { estudos sobre a } \\
\text { avaliação da } \\
\text { talassoterapia e/ou a } \\
\text { balneoterapia como } \\
\text { abordagem terapêutica } \\
\text { para fibromialgia. }\end{array}$ & $\begin{array}{c}\text { Ainda há controvérsias sobre a } \\
\text { eficácia das terapias. }\end{array}$ \\
\hline $\begin{array}{l}\text { Gusi et al; } \\
\quad 2006\end{array}$ & $\begin{array}{l}\mathrm{N}=34 \text { (mulheres; } \\
\text { grupo experimental } \\
(\mathrm{N}=17) \text { e grupo } \\
\text { controle }(\mathrm{N}=17))\end{array}$ & $\begin{array}{l}\text { Ensaio Clínico. Instrumentos. } \\
\text { Instrumentos: Torque máximo; } \\
\text { dinamômetro isocinético; } \\
\text { Questionário de qualidade de } \\
\text { vida (EQ5D); Escala Visual } \\
\text { Analógica da Dor (VAS). }\end{array}$ & $\begin{array}{l}\text { Intervenção com } \\
\text { hidroginásticalh, } 3 \mathrm{x} \\
\text { semanais, por } 12 \\
\text { semanas. }\end{array}$ & $\begin{array}{l}\text { Ambos os grupos melhora da } \\
\text { força de extensores e flexores, } \\
\text { qualidade de vida (exceto nas } \\
\text { atividades diárias). Grupo } \\
\text { experimental diminuiu a dor e a } \\
\text { ansiedade de depressão retornou a } \\
\text { valores base pós destreino. }\end{array}$ \\
\hline $\begin{array}{l}\text { Andrade CP } \\
\text { et al; } 2018\end{array}$ & $\begin{array}{l}\mathrm{N}=54 \text { (mulheres; } \\
\text { grupo intervenção e } \\
\text { destreino (GI; } \\
\mathrm{N}=27 \text { ) e grupo } \\
\text { controle (GC; } \\
\mathrm{N}=27) \text { ). }\end{array}$ & $\begin{array}{c}\text { Ensaio Clínico Randomizado. } \\
\text { Instrumentos: FIQ, SF-36, BDI, } \\
\text { Beck's Anxiety Inventory (BAI), } \\
\text { PSQI, Avaliação da composição } \\
\text { corporal, protocolo CPET, IPAQ; } \\
\text { VO2 absoluto e VO2 em relação } \\
\text { à TBM. }\end{array}$ & $\begin{array}{l}\text { Grupo Experimental: } 16 \\
\text { semanas, } 2 \text { vezes } \\
\text { semanais e controle } \\
\text { recebeu instruções para a } \\
\text { manutenção das } \\
\text { atividades de vida. }\end{array}$ & $\begin{array}{c}\text { APT contribuiu tanto para } \\
\text { aumentar o VO2 no VAT e no } \\
\text { CPET de pico, e melhorou os } \\
\text { sintomas clínicos. No destreino, } \\
\text { essas variáveis foram reduzidas } \\
\text { perto da linha de base. }\end{array}$ \\
\hline $\begin{array}{l}\text { Ardiç F et al; } \\
2007\end{array}$ & $\begin{array}{l}\mathrm{N}=24 \text { (grupo } \\
\text { intervenção }(\mathrm{N}=12) \\
\text { e Grupo sem } \\
\text { intervenção }(\mathrm{N}= \\
\text { 12), Grupo controle } \\
\text { sem patologia } \\
(\mathrm{N}=10)) .\end{array}$ & $\begin{array}{l}\text { Ensaio clínico randomizado. } \\
\text { Instrumentos: VAS; FIQ; BDI; } \\
\text { níveis de PGE2; número Tender } \\
\text { points. }\end{array}$ & $\begin{array}{l}\text { Intervenção de } 20 \\
\text { minutos de banho, } 5 \text { dias } \\
\text { por semana, total de } 15 \\
\text { sessões, e controle não } \\
\text { realizou a atividade. }\end{array}$ & $\begin{array}{c}\text { Balneoterapia é eficaz no } \\
\text { tratamento em pacientes com } \\
\text { fibromialgia, aliviando os } \\
\text { sintomas clínicos e possivelmente } \\
\text { influenciado os mediadores } \\
\text { inflamatórios. }\end{array}$ \\
\hline $\begin{array}{l}\text { Latorre PA et } \\
\text { al; } 2013\end{array}$ & $\begin{array}{l}\mathrm{N}=72 \text { (mulheres; } \\
\text { com fibromialgia; } \\
\text { grupo Intervenção } \\
(\mathrm{N}=42) \text { e Controle } \\
\quad(\mathrm{N}=30)) .\end{array}$ & $\begin{array}{l}\text { Ensaio clínico randomizado. } \\
\text { Instrumentos: VAS; algômetro, } \\
\text { capacidade funcional (força da } \\
\text { perna, dinamometria de preensão } \\
\text { manual, flexibilidade, agilidade, } \\
\text { equilíbrio, resistência aeróbica), } \\
\text { composição corporal, FIQ e SF- } \\
36 .\end{array}$ & $\begin{array}{l}\text { Intervenção com } \\
\text { exercícios na piscina } \\
\text { realizados } 2 \text { vezes na } \\
\text { semana e } 1 \text { vez por } \\
\text { semana exercícios na terra }\end{array}$ & $\begin{array}{l}\text { O grupo que realizou a } \\
\text { intervenção teve redução do } \\
\text { quadro de dor, impactando na } \\
\text { melhora da doença e na } \\
\text { capacidade funcional, além da } \\
\text { melhora da qualidade de vida de } \\
\text { mulheres com fibromialgia }\end{array}$ \\
\hline $\begin{array}{l}\text { Sevimli D et } \\
\text { al; } 201\end{array}$ & $\begin{array}{l}\mathrm{N}=75 \text { (mulheres } \\
\text { com fibromialgia; } \\
\text { grupo exercícios } \\
\text { isométricos em casa } \\
\text { (ISSEP), exerc. } \\
\text { aeróbicos (AEP) e } \\
\text { exerc. aeróbico } \\
\text { aquáticos na piscina } \\
\text { (AAEP) }\end{array}$ & $\begin{array}{l}\text { Estudo observacional. } \\
\text { Instrumentos: VAS, FIQ, o teste } \\
\text { de caminhada de seis minutos } \\
\text { (TC6), SF-36, e BDI. }\end{array}$ & $\begin{array}{l}\text { Intervenção de três meses } \\
\text { usando ISSEP ( } 15 \text { min. } \\
\text { diários), AEP ( } 2 \text { vezes na } \\
\text { semana) e AAEP ( } 2 \text { vezes } \\
\text { na semana). }\end{array}$ & $\begin{array}{l}\text { Todos os grupos mostraram } \\
\text { melhorias significativas em todas } \\
\text { as variáveis, exceto os valores } \\
\text { médios de VAS e BDI em ISSEP. } \\
\text { O AAEP foi o tratamento mais } \\
\text { eficaz para mulheres com } \\
\text { fibromialgia. }\end{array}$ \\
\hline $\begin{array}{l}\text { Pérez de La } \\
\text { Cruz S; } \\
\text { Lambeck JA; } \\
2004\end{array}$ & $\mathrm{~N}=20$ (mulheres) & $\begin{array}{l}\text { Estudo piloto experimental. } \\
\text { Instrumentos: Questionário } \\
\text { sociodemográfico, VAS, SF-36. }\end{array}$ & $\begin{array}{l}\text { Intervenção com } 20 \\
\text { sessões de } 45 \text { minutos, } \\
\text { durante } 10 \text { semanas de } \\
\text { tratamento. }\end{array}$ & $\begin{array}{l}\text { O treinamento de } \mathrm{AI} \text { CHI na água } \\
\text { pode contribuir para melhora da } \\
\text { saúde física e mental, e qualidade } \\
\text { de vida. }\end{array}$ \\
\hline $\begin{array}{l}\text { Franzen CG; } \\
\text { Ide MR; } 2004\end{array}$ & & Revisão de literatura & $\begin{array}{l}\text { Estudos sobre os } \\
\text { benefícios dos exercícios } \\
\text { aeróbicos aquáticos em } \\
\text { pacientes com } \\
\text { fibromialgia. }\end{array}$ & $\begin{array}{l}\text { Exercícios aeróbicos aquáticos } \\
\text { exercem efeito positivo na } \\
\text { qualidade de vida. }\end{array}$ \\
\hline
\end{tabular}




\begin{tabular}{|c|c|c|c|c|}
\hline $\begin{array}{l}\text { Gowans SA; } \\
\text { de Hueck A; } \\
\quad 2007\end{array}$ & & Revisão sistemática & $\begin{array}{l}\text { Seleção de análise de } 8 \\
\text { ensaios clínicos } \\
\text { randomizados }\end{array}$ & $\begin{array}{c}\text { Exercício na piscina demonstrou } \\
\text { melhorias no humor, assim como } \\
\text { na redução da ansiedade e } \\
\text { depressão. }\end{array}$ \\
\hline $\begin{array}{l}\text { Izquierdo D, } \\
\text { \& Arrese A.; } \\
\quad 2008\end{array}$ & $\begin{array}{l}\mathrm{N}=53 \text { (mulheres; } \\
\text { grupo intervenção } \\
(\mathrm{N}=29) \text { e controle } \\
\quad(\mathrm{N}=24)) .\end{array}$ & $\begin{array}{l}\text { Ensaio Clínico Randomizado. } \\
\text { Instrumentos: Questionário sobre } \\
\text { estado de saúde, FIQ, } \\
\text { Localização dos Tender points; } \\
\text { PSQI; testes físicos; escala } \\
\text { psicológica (SAI), da função } \\
\text { cognitiva (Paced Auditory Serial } \\
\text { Addition Task); e adesão. }\end{array}$ & $\begin{array}{c}\text { Intervenção de } 16 \\
\text { semanas de treinamento } \\
\text { em piscina de água morna }\end{array}$ & $\begin{array}{l}\text { O treinamento foi eficaz para } \\
\text { diminuir Tender points e melhoria } \\
\text { da qualidade do sono, função } \\
\text { cognitiva e física, e estado de } \\
\text { saúde. Grupo controle não obteve } \\
\text { mudanças. O estudo teve boa } \\
\text { adesão dos participantes. }\end{array}$ \\
\hline $\begin{array}{l}\text { Andrade A et } \\
\text { al; } 2018\end{array}$ & & Revisão Sistemática & $\begin{array}{l}22 \text { estudos de intervenção } \\
\text { com treinamento de força }\end{array}$ & $\begin{array}{l}\text { O treinamento reduz o número de } \\
\text { Tiger points, fadiga, depressão, } \\
\text { ansiedade, melhora a qualidade } \\
\text { do sono e de vida. O treinamento } \\
\text { de força é benéfico para o } \\
\text { tratamento da fibromialgia. }\end{array}$ \\
\hline
\end{tabular}

Fonte: Autores.

\section{Resultados e Discussão}

A partir desta busca a revisão foi estruturada e será apresentada da seguinte forma: Fisiopatologia, Principais instrumentos de avaliação e Efeito de intervenções aquáticas sobre os sintomas da Fibromialgia, subsequente a discussão.

\section{Fisiopatologia}

O modelo fisiopatológico que melhor descreve a fibromialgia parte da observação de que os aparecimentos dos sintomas dolorosos ocorrem de forma geralmente espontânea, simétrica e num sentido craniocaudal, sugerindo como causa para a síndrome disfunções no sistema nervoso central (Martinez et al., 2017; Wolfe et al., 2010).

Os mecanismos mais aceitos para o entendimento fisiopatológico da fibromialgia no momento envolvem o desequilíbrio entre a percepção dolorosa e os mecanismos de modulação dessas vias aferentes. Níveis elevados de substância P em líquor e níveis reduzidos de serotonina e seus precursores em líquor, soro e plaquetas são sugestivos desses desequilíbrios, uma vez que a substância P é mediadora das vias aferentes enquanto a serotonina medeia a inibição da dor (Talotta et al., 2017; Wolfe et al., 2010). As alterações do metabolismo da serotonina implicam na redução da atividade do Sistema Inibidor de Dor, com uma consequente elevação da resposta dolorosa frente a estímulos algiogênicos ou mesmo o aparecimento de dor espontânea (Theoharides et al.,2015; Heymann et al., 2017). Outra explicação para a alteração da atividade da serotonina seriam alterações genéticas dos receptores de serotonina, o que pode explicar também o agrupamento familiar desses pacientes (Talotta et al., 2017).

Essas alterações seriam responsáveis por outros achados habituais entre portadores de fibromialgia como a alteração do sono, transtornos ansiosos e outras síndromes disfuncionais associadas à ativação simpática, onde se incluem a cefaleia tensional, cólon irritável, dor torácica atípica e síndrome dismenorréica. Estudos clínicos de pacientes com fibromialgia mostram que alterações do sono são intimamente relacionadas com a sintomatologia somática, assim quanto pior o sono, maior o número de pontos dolorosos e em pacientes com fibromialgia. As alterações do sono também foram associadas à cronificação de queixas dolorosas (Heymann et al., 2017; Talotta et al., 2017; Theoharides et al., 2015).

\section{Principais instrumentos de avaliação dos sintomas da fibromialgia}

A literatura destaca que os instrumentos mais utilizados para a avaliação dos sintomas da fibromialgia são 
relacionados à intensidade da dor e ao impacto sobre a qualidade de vida dos pacientes (Martinez et al., 2017; Bidonde et al., 2014; Heymann et al., 2017).

Em relação à dor avalia-se a presença de dor e a sua intensidade. A presença da dor difusa associada à pressão em 11 de 18 pontos anatômicos dolorosos (tender points) é um indicativo de resposta positiva para fibromialgia. Esta avaliação é feita através de palpação ou seringas de pressão (Heymann et al., 2017).

Os pontos dolorosos podem ser úteis no diagnóstico da fibromialgia quando avaliados em conjunto com outros distúrbios funcionais contemplados nos critérios de 2010. Sua contagem pode se correlacionar com a intensidade de alguns sintomas, particularmente de estresse emocional (Heymann et al., 2017; Camargo, Moser, \& Bastos, 2009).

A avaliação da intensidade da dor é realizada por meio da Escala Visual Analógica da Dor (EVA). Esta escala varia de zero a $10 \mathrm{~cm}$, na qual o zero significa ausência de dor ou desconforto, e 10 o máximo de dor experimentada pelo paciente (Heymann et al., 2017; Camargo et al., 2009).

Quanto à avaliação do impacto provocado pela fibromialgia na qualidade de vida dos pacientes, os instrumentos mais utilizados são os questionários Fibromyalgia Impact Questionnaire (FIQ) e Medical Outcomes Study 36-Form Health Survey (SF36) e a escala de Beck Depression Inventory (BDI) para investigar a depressão (Heymann et al., 2017; Bidonde et al., 2014; Camargo et al., 2009).

O questionário FIQ leva em consideração as dimensões da vida de um indivíduo que podem ser afetadas pela síndrome, ou seja, a dimensão física associada à sua capacidade funcional, ao seu estado de saúde mental e aos sentimentos de bem-estar ou de dor. Revela ser um instrumento de fácil compressão e aplicação; é composto por 19 questões, e quanto maior o escore final maior o impacto da fibromialgia na qualidade de vida (Heymann et al., 2017).

A escala de SF-36 se destaca devido a sua forte presença na literatura, tanto em estudos descritivos, quanto em avaliações clínicas. De fato, o questionário SF-36 é uma das escalas genéricas mais recomendadas em estudos sobre dor ou distúrbios músculo esqueléticos (Heymann et al., 2017; Bidonde et al., 2014; Camargo et al., 2009; Ware, 2000).

O SF-36 é um instrumento de avaliação da qualidade de vida, de fácil administração e compreensão. Consiste em um questionário multidimensional formado por 36 itens, englobados em oito escalas ou domínios, que são: capacidade funcional, aspectos físicos, dor, estado geral da saúde, vitalidade, aspectos sociais, aspectos emocionais e saúde mental. Apresenta um escore final de 0 (zero) à 100 (obtido por meio de cálculo do Raw Scale), onde o zero corresponde ao pior estado geral de saúde e o 100 corresponde ao melhor estado de saúde (Ware, 2000).

A escala BDI é um instrumento que avalia a depressão em pacientes com doenças crônicas. A depressão pode indicar um estado anormal de humor quanto a um sintoma, uma síndrome ou uma doença. O questionário é composto de diversos itens relacionados aos sintomas depressivos como desesperança, irritabilidade e cognições como culpa ou sentimentos de estar sendo punido, assim como sintomas físicos como fadiga e perda de peso. Escores de 0 a 3 são atribuídos para 21 questões e a soma dos valores traça o perfil de humor do sujeito. Quanto maior o valor, maior a severidade dos sintomas depressivos (Heymann et al., 2017; Camargo et al., 2009).

\section{Efeito de intervenções aquáticas sobre os sintomas da Fibromialgia}

Utilizando os instrumentos de avaliação descritos anteriormente diversos autores apresentam resultados favoráveis da aplicação de intervenções com exercícios aquáticos no tratamento dos sintomas da fibromialgia.

Um estudo avaliou os efeitos de 2 sessões por semana durante 16 semanas de exercícios em água morna sobre a dor e a função cognitiva de mulheres com fibromialgia. As participantes que realizaram o treinamento tiveram uma diminuição do número de pontos de dor (tender points), aumento do limiar de dor por pressão e a diminuição do impacto na qualidade de vida, comparadas com o grupo controle. Apresentaram também significativas melhoras nos escores de testes cognitivos como 
os testes de velocidade de digitação, velocidade de processamento de informações, acuidade visual e auditiva comparados com seus valores pré-intervenção. Os autores atribuem essa melhora no desempenho cognitivo a diminuição dos pontos de dor e ao aumento do limiar de dor e chamam a atenção para o fato de como a dor provocada pela fibromialgia impacta não somente nas funções físicas, mas também, em atividades intelectuais interferindo na concentração e atenção dos pacientes (Izquierdo \& Arrese, 2007).

Em uma revisão sistemática foi constatado que programas de intervenções aquáticas, que apresentaram resultados significativos na diminuição de dor e melhora nos parâmetros de qualidade de vida em ensaios clínicos randomizados, são compostos frequentemente por exercícios aeróbicos, de força, flexibilidade e relaxamento. A intensidade dos exercícios variando entre $60 \%$ a $80 \%$ da frequência cardíaca máxima e sessões com duração média de 60 minutos, de 2 a 3 vezes por semana (Perraton, Machotka \& Kumar, 2009).

Letieri et al. (2013) buscaram analisar os efeitos do tratamento com hidrocinesioterapia na qualidade de vida, percepção de dor e gravidade de episódios depressivos em pacientes com fibromialgia. Os sujeitos foram submetidos a um tratamento hidrocinesioterápico em uma piscina aquecida ao longo de 15 semanas e avaliados através das escalas EVA, FIQ, e BDI. Os exercícios subaquáticos foram: de condicionamento cardiovascular, de força, de mobilidade, de coordenação, de equilíbrio, de alongamento e de relaxamento muscular. Foram observadas melhorias estatisticamente significativas na percepção da intensidade da dor, na qualidade de vida e nos sintomas de depressão favoráveis ao grupo hidrocinesioterapia comparado ao grupo controle.

Outro estudo (Assis et al., 2006) comparou a efetividade do treinamento de Deep Water com o treinamento de caminhada e corrida durante 15 semanas 3 vezes por semana sobre os sintomas da fibromialgia. Ambos os grupos obtiveram melhoras significativas na qualidade de vida tanto nos parâmetros físicos (dor) quanto emocionais (depressão e ansiedade). Estes resultados demonstraram que a corrida em piscina funda pode ser uma opção segura e viável de exercício sem impacto para pacientes com fibromialgia que tenham problemas de adaptação a exercícios em terra como, por exemplo, risco de quedas ou algum tipo de limitação articular.

Andrade et al., (2008) ao avaliar a efetividade sobre os sintomas da fibromialgia de exercícios aeróbicos na piscina comparados com exercícios aeróbicos no mar durante 12 semanas, 3 vezes por semana, puderam observar que houve melhoras significativas em todos os parâmetros clínicos em ambos os grupos: Intensidade da dor, $\mathrm{n}^{\circ}$ de pontos de dor, capacidade funcional, qualidade do sono e depressão.

Um estudo com 12 semanas de treinamento de hidroginástica com mulheres com fibromialgia resultou na diminuição da intensidade da dor e na melhora significativa em parâmetros de qualidade de vida e força muscular de membros inferiores. Ao reavaliar as pacientes 24 semanas depois, observaram que todos os parâmetros físicos retornaram aos níveis prétreinamento, mas interessantemente os níveis de ansiedade e depressão permaneceram nos alcançados pós-treinamento. Essas melhorias e manutenção nos níveis de ansiedade, depressão e na qualidade de vida podem ser parcialmente explicados pela interação com o professor e a oportunidade de compartilhar experiências de vida com outros participantes com fibromialgia durante os treinos em grupo (Gusi, Carus, Häkkinen, Häkkinen, \& Ortega, 2006). Resultados semelhantes foram obtidos por Andrade e colaboradores (2018), após um treinamento aquático de 16 semanas. Houve melhoras em parâmetros físicos e diminuição da dor, os quais retornaram aos valores de base após subsequentes 16 semanas de destreino. Estes achados vêm a reforçar a importância da adesão dos pacientes com fibromialgia aos programas terapêuticos para que os avanços na melhora dos sintomas alcançados em um período de intervenção se mantenham.

Outro mecanismo que tenta explicar a melhora clínica de pacientes com fibromialgia quando submetidos a intervenções aquáticas é sua possível ação sobre os mediadores inflamatórios. Ardiç et al., (2007) estudaram a influência da balneoterapia realizada durante três semanas sobre os níveis séricos de marcadores inflamatórios em pacientes com 
fibromialgia. Todos os parâmetros bioquímicos e clínicos foram avaliados antes e após a intervenção. Os autores observaram, além da melhora nos escores dos parâmetros clínicos (diminuição do número de pontos de dor, EVA, FIQ, BDI) o grupo que recebeu o tratamento teve uma diminuição dos marcadores de inflamação PGE2, IL-1 e LTB 4, comparados ao grupo controle, sugerindo a influência de agentes inflamatórios nos sintomas da fibromialgia e que a diminuição desses marcadores, provocada pela intervenção aquática possivelmente teria contribuído para a melhora dos sintomas.

Latorre et al. (2013) constataram que uma intervenção de 24 semanas composta por 2 sessões de exercícios aquáticos e 1 sessão de exercícios em terra por semana é capaz de reduzir a dor e o impacto da doença sobre a qualidade de vida de pacientes com fibromialgia. $\mathrm{O}$ efeito da intervenção neste estudo também sugere que pode existir uma relação positiva entre o treinamento dentro d'água e na terra, utilizando exercícios de força muscular, resistência aeróbica e flexibilidade, realizados em conjunto nestes meios no mesmo programa de tratamento.

Em estudo comparando intervenções de exercícios aeróbicos realizados na água com exercícios aeróbicos em terra foi constatado que ambas intervenções são eficazes para a diminuição da dor, melhora da qualidade de vida e diminuição de índices de depressão em pacientes com fibromialgia. Na comparação dos resultados pode-se observar uma superioridade dos resultados dos exercícios aquáticos, o que os autores atribuem a possibilidade de haver menores forças de impacto nas articulações e para o fato de que a maioria dos movimentos serem com contrações concêntricas (Sevimli, Kozanoglu, Guzel, \& Doganay, 2015).

Pérez de la Cruz \& Lambeck (2018) avaliaram os efeitos de um programa terapêutico utilizando a técnica Ai Chi, 2 vezes por semana, durante 10 semanas, sobre a qualidade de vida relacionada à saúde de indivíduos com fibromialgia. Ai-Chi é um método terapêutico realizado em uma piscina com água aquecida, com profundidade em torno de 1 metro, e consiste em uma sequência de movimentos lentos com dificuldade progressiva, combinados com exercícios de respiração profunda. Foram encontradas melhorias significativas em parâmetros como percepção da dor, vitalidade, saúde mental, bem como, uma melhora global na qualidade de vida, comparadas com os valores pré-intervenção.

\section{Discussão}

O presente estudo vem reforçar as evidências de que intervenções em ambiente aquático são alternativas seguras e eficientes no manejo dos sintomas da fibromialgia. Os achados dos estudos apresentados nesta revisão demonstram melhoras significativas em parâmetros que incluem aspectos relacionados à saúde física e psicológica de pacientes com fibromialgia.

Em uma revisão sistemática (Bidonde et al., 2014), afirmam que o sucesso na melhora dos sintomas da fibromialgia em tratamentos que utilizam como recurso terapias não medicamentosas é essencial para que os indivíduos sejam encorajados ao tratamento contínuo e ininterrupto. No que diz respeito aos métodos que incluem práticas físicas em meio aquático, o controle de variáveis como volume e intensidade é fundamental, pois assim é possível ajustar a intervenção necessária para promover adaptações positivas e contribuir para o maior esclarecimento de como estas podem ter um efeito duradouro (Bidonde et al., 2014).

Os benefícios de intervenções aquáticas para pacientes com fibromialgia advêm da combinação das propriedades biofísicas da água: flutuabilidade, gradiente de pressão hidrostática, viscosidade e temperatura controlada com os efeitos dos exercícios (Pérez de la Cruz \& Lambeck, 2018; Bidonde et al., 2014; Gowans \& De Hueck, 2007; Franzen \& Ide, 2004).

A flutuabilidade na água tem o potencial de limitar substancialmente a tensão nas articulações durante o exercício. Essa redução no estresse articular permite correr e saltar em imersão, o que não seria tolerado em terra por pessoas com algum tipo de problema articular ou sobrepeso. Além disso, um amplo repertório de movimentos é possível, propiciando estímulos de propriocepção e equilíbrio corporal (Pérez de la Cruz \& Lambeck, 2018; Gowans \& De Hueck, 2007; Franzen \& Ide, 2004).

Além do alívio mecânico, a temperatura aquecida da água fornece tratamento imediato para dor muscular, espasmo ou rigidez que podem limitar a tolerância do exercício em terra. Também propicia um aumento nos estímulos sensoriais, 
competindo com os estímulos dolorosos. A água aquecida tem uma ação vasodilatadora propiciando o aumento do suprimento de sangue aos músculos, o que pode contribuir para a remoção de mediadores álgicos ao exercício (Pérez de la Cruz \& Lambeck, 2018; Bidonde et al., 2014; Gowans \& De Hueck, 2007; Franzen \& Ide, 2004).

Outra hipótese para as melhorias observadas no limiar de dor e qualidade de vida, decorrentes de intervenções com exercícios aquáticos, podem ser atribuídas a um aumento da atividade de mecanismos analgésicos endógenos promovendo uma diminuição da atividade simpática. Alguns mecanismos periféricos, como um aumento da densidade capilar da musculatura esquelética e diminuição da susceptibilidade a microtraumas musculares também podem ser capazes de melhorar a sintomatologia dolorosa (Izquierdo \& Arrese, 2008; Izquierdo \& Arrese, 2007).

A imersão na água apresenta a particularidade que, através do controle da velocidade dos movimentos, aumentando ou diminuindo, pode-se manipular a intensidade do exercício e assim direcionar o treinamento para que gere determinadas adaptações, como por exemplo, condicionamento aeróbico, de força e resistência muscular (Letieri et al., 2013).

A literatura destaca que as intervenções aquáticas podem desenvolver efeitos benéficos sobre componentes psicológicos de pacientes com fibromialgia diminuindo níveis de depressão e ansiedade. Porém, os pacientes com fibromialgia têm dificuldades para aderir aos exercícios físicos (Andrade et al., 2018). O que pode contribuir nesses aspectos, é o fato de as atividades serem desenvolvidas, normalmente, em grupos, o que possibilita a interação e a troca de experiências entre pessoas que enfrentam os mesmos desafios impostos pela doença, o que parece contribuir como um fator anímico, aumentando a motivação e o fortalecimento da adesão aos tratamentos (Pérez de la Cruz \& Lambeck, 2018; Andrade et al., 2018; Bidonde et al., 2014).

A adesão dos pacientes com fibromialgia aos programas terapêuticos é um fator determinante para que as possíveis melhoras dos sintomas, alcançadas devido à prática de intervenções aquáticas, se mantenham, pois estudos mostraram que, após um período de destreino, melhoras obtidas em parâmetros da doença se perdem retornando aos valores de base (Andrade et al., 2018; Gusi et al., 2006).

\section{Conclusão}

Esta revisão de literatura possibilitou caracterizar as produções científicas com os seus aspectos metodológicos e resultados sobre as diferentes intervenções de exercícios aquáticos utilizados na síndrome da fibromialgia. Conclui-se a importância das intervenções aquáticas como recursos terapêuticos eficientes para o tratamento da dor e melhora da qualidade de vida de pessoas com fibromialgia.

Mesmo constatando algumas lacunas nos estudos de intervenção aquática retratados na literatura. Destaca-se a importância da realização de novos estudos, com maior rigor metodológico, para aferir com maior precisão, as relações de causa e efeito sobre os sintomas da fibromialgia.

\section{Referências}

Andrade, A., Steffens, R., Sieczkowska, S., Peyré Tartaruga, L., \& Vilarino, G. (2018). A systematic review of the effects of strength training in patients with fibromyalgia: clinical outcomes and design considerations. Advances in Rheumatology (London, England), 58(1), 36. https://doi.org/10.1186/s42358-0180033-9

Andrade, C., Zamunér, A., Forti, M., Tamburús, N., \& Silva, E. (2018). Effects of aquatic training and detraining on women with fibromyalgia: Controlled randomized clinical trial. European Journal of Physical and Rehabilitation Medicine, 55(1), 79-88. https://doi.org/10.23736/S1973-9087.18.05041-4

Ardiç, F., Merih, Ö., Hülya, Â., Rota, S., Çubukçu, D., \& Gökgöz, A. (2007). Efects of balneotherapy on serum IL-1 , PGE2 and LTB4 levels in fibromyalgia patients. Rheumatology International, 27(1), 441-446. https://doi.org/10.1007/s00296-006-0237-X

Assis, M. R., Silva, L. E., Alves, A. M. B., Pessanha, A. P., Valim, V., Feldman, D., ... Natour, J. (2006). A randomized controlled trial of deep water running: clinical effectiveness of aquatic exercise to treat fibromyalgia. Arthritis and Rheumatism, 55(1), 57-65. https://doi.org/10.1002/art.21693 
Bidonde, J., Busch, A. J., Webber, S. C., Schachter, C. L., Danyliw, A., Overend, T. J., ... Rader, T. (2014). Aquatic exercise training for fibromyalgia. The Cochrane Database of Systematic Reviews, 1(10), 1-173. https://doi.org/10.1002/14651858.CD011336

Camargo, R. S., Moser, A. D. D. L., \& Bastos, L. C. (2009). Abordagem dos métodos avaliativos em fibromialgia e dor crônica aplicada à tecnologia da informação: Revisão da literatura em periódicos, entre 1998 e 2008. Revista Brasileira de Reumatologia, 49(4), 431-446. https://doi.org/10.1590/S048250042009000400009

De Andrade, S. C., de Carvalho, R. F. P. P., Soares, A. S., de Abreu Freitas, R. P., de Medeiros Guerra, L. M., \& Vilar, M. J. (2008). Thalassotherapy for fibromyalgia: a randomized controlled trial comparing aquatic exercises in sea water and water pool. Rheumatology International, 29(2), 147-152. https://doi.org/10.1007/s00296-008-0644-2

Franzen, C. G., \& Ide, M. R. (2004). Influência do exercício aeróbico aquático na qualidade de vida de pacientes com fibromialgia: revisão narrativa. Arquivos de Ciências Saúde da UNIPAR, 8(1), 55-62. Retrieved from https://pesquisa.bvsalud.org/portal/resource/pt/lil-462600

Gowans, S. E., \& DeHueck, A. (2007). Pool exercise for individuals with fibromyalgia. Current Opinion in Rheumatology, 19(2), 168-173. https://doi.org/10.1097/BOR.0b013e3280327944

Gusi, N., Carus, P., Häkkinen, A., Häkkinen, K., \& Ortega, A. (2006). Exercise in waist-high warm water decreases pain and improves health-related quality of life and strength in the lower extremities in women with fibromyalgia. Arthritis Care and Research, 55(1), 66-73. https://doi.org/10.1002/art.21718

Heymann, R. E., Paiva, E. S., Martinez, J. E., Helfenstein, M., Rezende, M. C., Provenza, J. R., ... Souza, E. J. R. (2017). Novas diretrizes para o diagnóstico da fibromialgia. Revista Brasileira de Reumatologia, 57(S 2), 467-476. https://doi.org/10.1016/j.rbr.2017.05.006

Izquierdo, D., \& Arrese, A. (2007). Exercise in warm water decreases pain and improves cognitive function in middle-aged women with fibromyalgia. Clinical and Experimental Rheumatology, 25(6), 823-830.

Izquierdo, D., \& Arrese A. (2008). Assessment of the Effects of Aquatic Therapy on Global Symptomatology in Patients With Fibromyalgia Syndrome: A Randomized Controlled Trial. Archives of Physical Medicine and Rehabilitation, 89(12), 2250-2257. https://doi.org/10.1016/j.apmr.2008.03.026

Latorre, P. A., Santos, M. A., Heredia-Jiménez, J. M., Delgado-Fernández, M., Soto, V. M., Mañas, A., \& Carbonell-Baeza, A. (2013). Effect of a 24-week physical training programme (in water and on land) on pain, functional capacity, body composition and quality of life in women with fibromyalgia. Clinical and Experimental Rheumatology, 31(SUPPL.79), 72-80.

Kümpel C, Porto E, Silva K, Aguiar S, Lima P, Campos G., ...Castro A.. (2020). Estudo comparativo dos efeitos da hidroterapia e método Pilates sobre a capacidade funcional de pacientes portadores de fibromialgia. Acta Fisiatrica ;27(2), 64-70.

Letieri, R. V., Furtado, G. E., Letieri, M., Góes, S. M., Borba Pinheiro, C. J., Veronez, S. O., ... Dantas, E. M. (2013). Dor, qualidade de vida, autopercepção de saúde e depressão de pacientes com fibromialgia, tratados com hidrocinesioterapia. Revista Brasileira de Reumatologia, 53(6), 494-500. https://doi.org/10.1016/j.rbr.2013.04.004

Martinez, J. E., Paiva, E. S., Rezende, M. C., Heymann, R. E., Helfenstein, M., Ranzolin, A., ... Assis, M. R. de. (2017). EpiFibro (Brazilian Fibromyalgia Registry): data on the ACR classification and diagnostic preliminary criteria fulfillment and the follow-up evaluation. Revista Brasileira de Reumatologia, 57(2), 129-133. https://doi.org/10.1016/j.rbr.2016.07.003

Pérez de la Cruz, S., \& Lambeck, J. (2018). A new approach towards improved quality of life in fibromyalgia: a pilot study on the effects of an aquatic Ai Chi program. International Journal of Rheumatic Diseases, 21(8), 1525-1532. https://doi.org/10.1111/1756-185X.12930

Perraton, L., Machotka, Z., \& Kumar, S. (2009). Components of effective randomized controlled trials of hydrotherapy programs for fibromyalgia syndrome: A systematic review. Journal of Pain Research, 2, 165-173. https://doi.org/10.2147/jpr.s8052

Sevimli, D., Kozanoglu, E., Guzel, R., \& Doganay, A. (2015). The effects of aquatic, isometric strength-stretching and aerobic exercise on physical and psychological parameters of female patients with fibromyalgia syndrome. Journal of Physical Therapy Science, 27(6), 1781-1786. https://doi.org/10.1589/jpts.27.1781

Souza, J \& Perissinotti, D. (2018). Prevalence of fibromyalgia in Brazil - a population based study with secondary data of the study on chronic pain prevalence in Brazil. Brazilian Journal of Pain, 1(4), 345-348. https://doi.org/10.5935/2595-0118.20180065.

Talotta, R., Bazzichi, L., Di Franco, M., Casale, R., Batticciotto, A., Gerardi, M. C., \& Sarzi-Puttini, P. (2017). One year in review 2017: Fibromyalgia. Clinical and Experimental Rheumatology, 35, 6-12.

Theoharides, T. C., Tsilioni, I., Arbetman, L., Panagiotidou, S., Stewart, J. M., Gleason, R. M., \& Russell, I. J. (2015). Fibromyalgia syndrome in need of effective treatments. Journal of Pharmacology and Experimental Therapeutics, 355(2), 255-263. https://doi.org/10.1124/jpet.115.227298

Vilaça, M. M. O., Lima, K. B. dos S., Alencar, E. V. M., Machado, K. da C., \& Machado, K. da C. (2020). New fibromyalgia treatment medicinal products. Research, Society and Development, 9(3), e24932308. https://doi.org/10.33448/rsd-v9i3.2308

Ware, J. E. (2000). SF-36 Health Survey update. Spine, 25(24), 3130-3139. https://doi.org/10.1097/00007632-200012150-00008

Wolfe, F., Clauw, D. J., Fitzcharles, M., Goldenberg, D. O. N. L., Katz, R. S., Mease, P, ... Winfield, J. B. (2010). The American College of Rheumatology Preliminary Diagnostic Criteria for Fibromyalgia and Measurement of Symptom Severity. Arthritis Care \& Research, 62(5), 600-610. https://doi.org/10.1002/acr.20140 